\title{
Influence of body size on platelet response to ticagrelor and prasugrel in patients with acute coronary syndromes
}

\author{
Gjin Ndrepepa ${ }^{1}$ (1) $\cdot$ Stefan Holdenrieder ${ }^{2}$ Isabell Bernlochner ${ }^{3,4} \cdot$ Adnan Kastrati $^{1,4}$
}

Received: 22 October 2021 / Accepted: 22 November 2021 / Published online: 1 December 2021

(c) The Author(s) 2021

Sirs:

The influence of body size on platelet response to ticagrelor and prasugrel remains poorly investigated. So far, limited evidence exists on the platelet response to prasugrel in relation to body size indices [1]. In regard to ticagrelor, the platelet response to this drug has been investigated only according to body mass index (BMI) and the data are controversial [2-4]. We undertook this study to assess the influence of body size indices on the platelet response to ticagrelor and prasugrel in patients with acute coronary syndromes (ACS).

This study included 598 patients with ACS who were platelet $\mathrm{P}_{2} \mathrm{Y}_{12}$ receptor inhibitor-naïve on admission and who underwent platelet function tests in the setting of Intracoronary Stenting and Antithrombotic Regimen: Rapid Early Action for Coronary Treatment (ISAR-REACT) 5 trial (Clinical Trial Registration: NCT01944800) at the Klinikum rechts der Isar and Deutsches Herzzentrum München, Germany. Patients with ACS planned to undergo an invasive treatment strategy were randomized to ticagrelor (loading dose of $180 \mathrm{mg}$ ) or prasugrel (loading dose of $60 \mathrm{mg}$ ) with 1:1 randomization ratio. Detailed inclusion/exclusion criteria are reported in the primary publication [5]. Venous whole blood was obtained from patients before and after study drug loading dose administration. Adenosine diphosphate

Gjin Ndrepepa

ndrepepa@dhm.mhn.de

1 Deutsches Herzzentrum München, Technical University of Munich, Lazarettstrasse 36, 80636 Munich, Germany

2 Institut Für Laboratoriumsmedizin, Deutsches Herzzentrum München, Technical University of Munich, Munich, Germany

3 Medizinische Klinik Und Poliklinik Innere Medizin I, Klinikum Rechts Der Isar, Technical University of Munich, Munich, Germany

4 German Center for Cardiovascular Research (DZHK), Partner Site Munich Heart Alliance, Munich, Germany
(ADP)-induced platelet aggregation values were measured using the Mulitplate ${ }^{\circledR}$ Analyzer (Roche Diagnostics, Switzerland). Platelet aggregation values were quantified as area under the curve of aggregation units (AU $\times$ min) [6].

Five body size indices were included in the current analysis: body weight (BW) in $\mathrm{kg}$; BMI calculated as weight $(\mathrm{kg}) /$ height $(\mathrm{m})^{2}$; body surface area (BSA) calculated as $\mathrm{BSA}=\left(\right.$ weight $[\mathrm{kg}]^{0.425} \times$ height $\left.[\mathrm{cm}]^{0.725}\right) \times 0.00$ 7184 [7]; lean body mass (LBM) calculated as, $\mathrm{LBM}=($ $1.1 \times$ weight $[\mathrm{kg}])-128 \times(\text { weight }[\mathrm{kg}] / \text { height }[\mathrm{cm}])^{2}$ in men and $\mathrm{LBM}=(1.07 \times$ weight $[\mathrm{kg}])-148 \times($ weight $[\mathrm{kg}] /$ height $[\mathrm{cm}])^{2}$ in women [8]; and blood volume (BV) calculated as, $\mathrm{BV}=\left(0.006012 \times\right.$ height $\left.[\text { inch }]^{3}\right) /(14.6 \times$ weight [pound] +604 in men and $\mathrm{BV}=\left(0.005835 \times{\left.\text { height }[\text { inch }]^{3}\right)}^{3}\right.$ / $(15 \times$ weight [pound] $)+183$ in women [9].

The primary outcome was ADP-induced platelet aggregation values within $24 \mathrm{~h}$ following loading dose of ticagrelor and prasugrel. Patients were categorized in subgroups according to tertiles of each body size index. Data are presented as medians with 25 th-75th percentiles, mean \pm standard deviation or counts (\%). Comparison between groups was performed using the Kruskal-Wallis rank-sum test. A two-sided $P<0.05$ was considered to indicate statistical significance. The study was approved by the Local Ethics Committee and conforms to the Declaration of Helsinki.

Baseline data are shown in Table 1. Baseline demographic, clinical and procedural characteristics were well balanced between patients in ticagrelor and prasugrel groups. All patients underwent percutaneous coronary intervention. Measurement of ADP-induced platelet aggregation was performed after a median [25th-75th percentiles] of 11.8 [5.4-16.9] hours after prasugrel loading and 11.8 [6.1-17.8] hours after ticagrelor loading $(P=0.299)$. ADP-induced platelet aggregation values at baseline and within $24 \mathrm{~h}$ after drug loading are shown in Table 2. Baseline ADP-induced platelet aggregation values did not differ significantly according to the tertiles of all body size indices in ticagrelor or prasugrel groups. A 
Table 1 Baseline characteristics

\begin{tabular}{|c|c|}
\hline Characteristic $(n=598)$ & Value \\
\hline Age (years) & $65.0[55.0-75.0]$ \\
\hline Women & $126(21.1)$ \\
\hline Diabetes mellitus & $129(21.6)$ \\
\hline Current smoking & $198(33.7)$ \\
\hline History of arterial hypertension & $431(72.6)$ \\
\hline History of hypercholesterolemia & $385(64.4)$ \\
\hline Previous myocardial infarction & $109(18.3)$ \\
\hline Previous percutaneous coronary intervention & $149(25.0)$ \\
\hline Previous coronary artery bypass surgery & $40(6.7)$ \\
\hline Systolic blood pressure (mmHg) & $150[130-163]$ \\
\hline Diastolic blood pressure $(\mathrm{mmHg})$ & 80 [75-90] \\
\hline Unstable angina & $64(10.7)$ \\
\hline Non-ST-segment elevation myocardial infarction & $254(42.5)$ \\
\hline ST-segment elevation myocardial infarction & $280(46.8)$ \\
\hline Coronary angiography & $598(100.0)$ \\
\hline Femoral artery access & $581(97.2)$ \\
\hline Radial artery access & $17(2.8)$ \\
\hline \multicolumn{2}{|l|}{ Extent of coronary artery disease } \\
\hline One-vessel disease & $145(24.2)$ \\
\hline Two-vessel disease & $172(28.8)$ \\
\hline Three vessel disease & $281(47.0)$ \\
\hline Left ventricular ejection fraction $(\%)$ & $48.7 \pm 10.1$ \\
\hline \multicolumn{2}{|l|}{ Post-procedural TIMI flow grade } \\
\hline 0 & $9(1.5)$ \\
\hline 1 & $1(0.2)$ \\
\hline 2 & $25(4.2)$ \\
\hline 3 & $563(94.1)$ \\
\hline Percutaneous coronary intervention & $598(100.0)$ \\
\hline Drug-eluting stent & $500(83.6)$ \\
\hline Bioresorbable vascular scaffold & $67(11.2)$ \\
\hline \multicolumn{2}{|l|}{ Periprocedural antithrombotic therapy } \\
\hline Aspirin & $559(93.4)$ \\
\hline Unfractionated heparin & $596(99.7)$ \\
\hline Low molecular weight heparin & $5(0.8)$ \\
\hline Bivalirudin & $1(0.2)$ \\
\hline Beta-blocking agents on admission & $180(30.1)$ \\
\hline Statins on admission & $182(30.4)$ \\
\hline Body weight (kg) & $81.0[72.0-93.0]$ \\
\hline Body mass index $\left(\mathrm{kg} / \mathrm{m}^{2}\right)$ & $26.9[24.7-29.7]$ \\
\hline Body surface area $\left(\mathrm{m}^{2}\right)$ & $1.97[1.83-2.10]$ \\
\hline Lean body mass $(\mathrm{kg})$ & $58.9[53.2-63.9]$ \\
\hline Blood volume (Liter) & $5.18[4.62-5.64]$ \\
\hline
\end{tabular}

Data are median with 25 th -75 th percentiles, mean \pm standard deviation or count $(\%)$

TIMI thrombolysis in myocardial infarction

strong platelet inhibition was observed in ticagrelor and prasugrel groups and the platelet inhibition was stronger with prasugrel than ticagrelor. The deeper platelet inhibition with prasugrel versus ticagrelor in all tertiles of body size indices supports the main findings of the primary trial [5]. In the ticagrelor-treated patients, the ADP-induced platelet aggregation values within $24 \mathrm{~h}$ following drug loading did not to differ according to tertiles of each body size index ( $P \geq 0.488$ for all comparisons). In the prasugrel-treated patients, the ADP-induced platelet aggregation values were higher in upper tertiles of BW, BSA, LBM and $\mathrm{BV}$, but not BMI. The level of statistical significance was achieved for BSA $(P=0.016), \mathrm{LBM}(P=0.042)$ and BV $(P=0.017$; Table 2).

The main findings of this study may be summarized as follows: (1) Platelet response to ticagrelor appears not to depend on the body size. ADP-induced platelet aggregation values within $24 \mathrm{~h}$ following ticagrelor loading did not differ according to tertiles of body size indices investigated in the current study. (2) Platelet response to prasugrel appears to differ according to body size indices with higher platelet aggregation values in upper tertiles in four of five body size indices investigated in this analysis. (3) Platelet response to ticagrelor or prasugrel appears not to differ according to BMI tertiles, the commonly used body size index in pharmacological and clinical studies involving antiplatelet drugs. Thus, BMI may be suboptimal to guide dosing of antiplatelet drugs based on the platelet response to a given drug. Jakubowski et al.[1] reported an inverse relationship between body size indices (BW, BMI and BSA) and response to clopidogrel or prasugrel. Notably, the body size was a determinant of exposure to active metabolites and residual platelet activity regardless of type and dose of thienopyridine and BW and BSA showed stronger correlations with platelet reactivity [1]. With regard to platelet response to ticagrelor in relation to body size indices, a study by Deharo et al. [2] reported that BMI did not impact platelet inhibition by ticagrelor in patients with ACS. The frequency of drug administration (twice daily for ticagrelor and once daily for prasugrel) may impact on the platelet response to ticagrelor or prasugrel according to the body size. The study has limitations mostly related to being a subgroup analysis and to not repeating the platelet inhibition tests during the study course.

In conclusion, body size appears to influence the platelet response to prasugrel but not to ticagrelor. Among body size indices investigated, higher values of BSA, LBM and BV but not BMI were associated with higher ADP-induced platelet aggregation values within $24 \mathrm{~h}$ following prasugrel loading. These data may serve to optimize dosing of antiplatelet drugs in patients with ACS. Future studies are needed to confirm these results and clarify how antiplatelet drug dosing could be adjusted according to the body size. 
Table 2 Platelet aggregation measurement at baseline and within $24 \mathrm{~h}$ after drug loading dose administration

\begin{tabular}{|c|c|c|c|c|c|c|c|c|}
\hline Body size & Ticagrelor $(n=2$ & & & $P$ value & Prasugrel $(n=30$ & & & $P$ value \\
\hline BW tertiles & $1(n=98)$ & $2(n=95)$ & $3(n=96)$ & & $1(n=109)$ & $2(n=109)$ & $3(n=91)$ & \\
\hline BW (kg) & $\begin{array}{l}70.0 \\
{[62.2-73.0]}\end{array}$ & $\begin{array}{l}81.0 \\
{[80.0-85.0]}\end{array}$ & $\begin{array}{l}96.0 \\
{[93.0-106.0]}\end{array}$ & $<0.001$ & $\begin{array}{l}69.0 \\
{[63.0-72.0]}\end{array}$ & $\begin{array}{l}82.0 \\
{[80.0-85.0]}\end{array}$ & $\begin{array}{l}100.0 \\
{[95.0-108.0]}\end{array}$ & $<0.001$ \\
\hline $\begin{array}{l}\text { Baseline ADP- } \\
\text { induced } \\
\text { platelet } \\
\text { aggrega- } \\
\text { tion values } \\
\text { (AU } \times \text { min) }\end{array}$ & 862 [607-1112] & $\begin{array}{l}832 \\
{[593-1134]}\end{array}$ & 798 [628-1122] & 0.941 & 832 [555-1102] & 833 [654-1124] & 845 [632-1005] & 0.751 \\
\hline $\begin{array}{l}\text { After drug } \\
\text { loading } \\
\text { dose ADP- } \\
\text { induced } \\
\text { platelet } \\
\text { aggrega- } \\
\text { tion values } \\
(\text { AU } \times \text { min })\end{array}$ & $\begin{array}{l}130 \\
{[73.5-198]}\end{array}$ & $\begin{array}{l}147 \\
{[84.5-230]}\end{array}$ & $\begin{array}{l}126 \\
{[75-197]}\end{array}$ & 0.488 & $\begin{array}{l}88 \\
{[48-149]}\end{array}$ & $\begin{array}{l}116 \\
{[64-173]}\end{array}$ & $\begin{array}{l}117 \\
{[63-199]}\end{array}$ & 0.054 \\
\hline BMI tertiles & $1(n=97)$ & $2(n=96)$ & $3(n=96)$ & & $1(n=104)$ & $2(n=102)$ & $3(n=103)$ & \\
\hline BMI $\left(\mathrm{kg} / \mathrm{m}^{2}\right)$ & $\begin{array}{l}23.8 \\
{[22.1-24.8]}\end{array}$ & $\begin{array}{l}26.8 \\
{[26.1-27.8]}\end{array}$ & $\begin{array}{l}31.1 \\
{[29.6-34.4]}\end{array}$ & $<0.001$ & $\begin{array}{l}23.7 \\
{[22.2-24.5]}\end{array}$ & $\begin{array}{l}27.1 \\
{[26.2-27.7]}\end{array}$ & $\begin{array}{l}31.4 \\
{[29.9-34.0]}\end{array}$ & $<0.001$ \\
\hline $\begin{array}{l}\text { Baseline ADP- } \\
\text { induced } \\
\text { platelet } \\
\text { aggrega- } \\
\text { tion values } \\
(\text { AU } \times \text { min })\end{array}$ & $\begin{array}{l}832 \\
{[608-1108]}\end{array}$ & $\begin{array}{l}867 \\
{[547-1144]}\end{array}$ & $\begin{array}{l}784 \\
{[634-1083]}\end{array}$ & 0.927 & $\begin{array}{l}833 \\
{[561-1105]}\end{array}$ & $\begin{array}{l}831 \\
{[638-1113]}\end{array}$ & 843 [636-1018] & 0.753 \\
\hline $\begin{array}{l}\text { After drug } \\
\text { loading } \\
\text { dose ADP- } \\
\text { induced } \\
\text { platelet } \\
\text { aggrega- } \\
\text { tion values } \\
(\text { AU } \times \text { min) }\end{array}$ & $\begin{array}{l}132 \\
{[77-212]}\end{array}$ & $\begin{array}{l}146 \\
{[86-221]}\end{array}$ & $\begin{array}{l}126 \\
{[68-195]}\end{array}$ & 0.664 & $\begin{array}{l}107 \\
{[58-169]}\end{array}$ & $\begin{array}{l}102 \\
{[59-180]}\end{array}$ & $\begin{array}{l}103 \\
{[60-160]}\end{array}$ & 0.988 \\
\hline BSA tertiles & $1(n=97)$ & $2(n=96)$ & $3(n=96)$ & & $1(n=103)$ & $2(n=103)$ & $3(n=103)$ & \\
\hline $\operatorname{BSA}\left(\mathrm{m}^{2}\right)$ & $\begin{array}{l}1.77 \\
{[1.69-1.83]}\end{array}$ & $\begin{array}{l}1.97 \\
{[1.93-2.01]}\end{array}$ & $\begin{array}{l}2.16 \\
{[2.10-2.25]}\end{array}$ & $<0.001$ & $\begin{array}{l}1.75 \\
{[1.67-1.82]}\end{array}$ & $\begin{array}{l}1.97 \\
{[1.93-2.01]}\end{array}$ & $\begin{array}{l}2.15 \\
{[2.11-2.25]}\end{array}$ & $<0.001$ \\
\hline $\begin{array}{l}\text { Baseline ADP- } \\
\text { induced } \\
\text { platelet } \\
\text { aggrega- } \\
\text { tion values } \\
\text { (AU } \times \text { min })\end{array}$ & 881 [607-1149] & 786 [603-1098] & 794 [625-1122] & 0.554 & 848 [621-1105] & 828 [590-1098] & 836 [636-1008] & 0.899 \\
\hline $\begin{array}{l}\text { After drug } \\
\text { loading } \\
\text { dose ADP- } \\
\text { induced } \\
\text { platelet } \\
\text { aggrega- } \\
\text { tion values } \\
(\text { AU } \times \text { min })\end{array}$ & $\begin{array}{l}130 \\
{[86-198]}\end{array}$ & $\begin{array}{l}144 \\
{[80-224]}\end{array}$ & $\begin{array}{l}126 \\
{[75.5-202]}\end{array}$ & 0.845 & $\begin{array}{l}87 \\
{[47-140]}\end{array}$ & $\begin{array}{l}119 \\
{[66-174]}\end{array}$ & $\begin{array}{l}116 \\
{[63-199]}\end{array}$ & 0.016 \\
\hline LBM tertiles & $1(n=99)$ & $2(n=94)$ & $3(n=96)$ & & $1(n=103)$ & $2(n=103)$ & $3(n=103)$ & \\
\hline LBM (kg) & $\begin{array}{l}51.8 \\
{[48.5-53.4]}\end{array}$ & $\begin{array}{l}58.9 \\
{[57.3-60.3]}\end{array}$ & $\begin{array}{l}66.1 \\
{[63.9-69.0]}\end{array}$ & $<0.001$ & $\begin{array}{l}50.0 \\
{[47.0-53.0]}\end{array}$ & $\begin{array}{l}59.0 \\
{[57.3-60.3]}\end{array}$ & $66.0[64.3-68.8]$ & $<0.001$ \\
\hline
\end{tabular}


Table 2 (continued)

\begin{tabular}{|c|c|c|c|c|c|c|c|c|}
\hline Body size & Ticagrelor $(n$ & 89) & & $P$ value & Prasugrel $(n=$ & & & $P$ value \\
\hline $\begin{array}{l}\text { Baseline ADP- } \\
\text { induced } \\
\text { platelet } \\
\text { aggrega- } \\
\text { tion values } \\
(\text { AU } \times \text { min })\end{array}$ & $\begin{array}{l}864 \\
{[596-1137]}\end{array}$ & $\begin{array}{l}798 \\
{[654-1115]}\end{array}$ & $\begin{array}{l}794 \\
{[600-1122]}\end{array}$ & 0.838 & $\begin{array}{l}857 \\
{[637-1118]}\end{array}$ & $\begin{array}{l}828 \\
{[569-1098]}\end{array}$ & $\begin{array}{l}822 \\
{[631-996]}\end{array}$ & 0.508 \\
\hline $\begin{array}{l}\text { After drug } \\
\text { loading } \\
\text { dose ADP- } \\
\text { induced } \\
\text { platelet } \\
\text { aggrega- } \\
\text { tion values } \\
(\text { AU } \times \text { min })\end{array}$ & $\begin{array}{l}129 \\
{[74-206]}\end{array}$ & $\begin{array}{l}144 \\
{[83-219]}\end{array}$ & $\begin{array}{l}135 \\
{[75.5-205]}\end{array}$ & 0.764 & $\begin{array}{l}88 \\
{[49-142]}\end{array}$ & $\begin{array}{l}119 \\
{[65-171]}\end{array}$ & $\begin{array}{l}117 \\
{[62.5-196]}\end{array}$ & 0.042 \\
\hline BV tertiles & $1(n=99)$ & $2(n=96)$ & $3(n=94)$ & & $1(n=103)$ & $2(n=103)$ & $3(n=103)$ & \\
\hline BV (Liter) & $\begin{array}{l}4.42 \\
{[4.15-4.65]}\end{array}$ & $\begin{array}{l}5.20 \\
{[5.05-5.32]}\end{array}$ & $\begin{array}{l}5.86 \\
{[5.63-6.17]}\end{array}$ & $<0.001$ & $\begin{array}{l}4.25 \\
{[3.82-4.60]}\end{array}$ & $\begin{array}{l}5.18 \\
{[4.99-5.31]}\end{array}$ & $\begin{array}{l}5.82 \\
{[5.64-6.14]}\end{array}$ & $<0.001$ \\
\hline $\begin{array}{l}\text { Baseline ADP- } \\
\text { induced } \\
\text { platelet } \\
\text { aggrega- } \\
\text { tion values } \\
\text { (AU } \times \text { min })\end{array}$ & $\begin{array}{l}880 \\
{[607-1148]}\end{array}$ & $\begin{array}{l}784 \\
{[628-1095]}\end{array}$ & $\begin{array}{l}810 \\
{[611-1125]}\end{array}$ & 0.551 & $\begin{array}{l}858 \\
{[644-1148]}\end{array}$ & $\begin{array}{l}809 \\
{[543-1060]}\end{array}$ & $\begin{array}{l}824 \\
{[636-999]}\end{array}$ & 0.209 \\
\hline $\begin{array}{l}\text { After drug } \\
\text { loading } \\
\text { dose ADP- } \\
\text { induced } \\
\text { platelet } \\
\text { aggrega- } \\
\text { tion values } \\
(\text { AU } \times \text { min })\end{array}$ & $\begin{array}{l}130 \\
{[74-208]}\end{array}$ & $\begin{array}{l}134 \\
{[80-208]}\end{array}$ & $\begin{array}{l}142 \\
{[79-206]}\end{array}$ & 0.858 & $\begin{array}{l}88 \\
{[44-140]}\end{array}$ & $\begin{array}{l}119 \\
{[65.5-174]}\end{array}$ & $\begin{array}{l}117 \\
{[63-196]}\end{array}$ & 0.017 \\
\hline
\end{tabular}

Data are median with 25 th-75th percentiles

$A D P$ adenosine diphosphate, $B M I$ body mass index, $B W$ body weight, $B S A$ body surface area, $B V$ blood volume, $L B M$ lean body mass

Funding Open Access funding enabled and organized by Projekt DEAL. Supported by a grant (FKZ 81X1600501) from the German Center for Cardiovascular Research and the Deutsches Herzzentrum München, Germany.

\section{Declarations}

Conflict of interest Dr. Bernlochner has received lecture fees from Sysmex Europe GmbH. Other authors have reported that they have no relationships relevant to the contents of this paper to disclose.

Open Access This article is licensed under a Creative Commons Attribution 4.0 International License, which permits use, sharing, adaptation, distribution and reproduction in any medium or format, as long as you give appropriate credit to the original author(s) and the source, provide a link to the Creative Commons licence, and indicate if changes were made. The images or other third party material in this article are included in the article's Creative Commons licence, unless indicated otherwise in a credit line to the material. If material is not included in the article's Creative Commons licence and your intended use is not permitted by statutory regulation or exceeds the permitted use, you will need to obtain permission directly from the copyright holder. To view a copy of this licence, visit http://creativecommons.org/licenses/by/4.0/.

\section{References}

1. Jakubowski JA, Angiolillo DJ, Zhou C, Small DS, Moser BA, Ten Berg JM, Brown PB, James S, Winters KJ, Erlinge D (2014) The influence of body size on the pharmacodynamic and pharmacokinetic response to clopidogrel and prasugrel: a retrospective analysis of the FEATHER study. Thromb Res 134:552-557

2. Deharo P, Pankert M, Bonnet G, Quilici J, Bassez C, Morange P, Alessi MC, Bonnet JL, Cuisset T (2014) Body mass index has no impact on platelet inhibition induced by ticagrelor after acute coronary syndrome, conversely to prasugrel. Int J Cardiol 176:1200-1202

3. Alexopoulos D, Xanthopoulou I, Storey RF, Bliden KP, Tantry US, Angiolillo DJ, Gurbel PA (2014) Platelet reactivity during 
ticagrelor maintenance therapy: a patient-level data meta-analysis. Am Heart J 168:530-536

4. Ono M, Chichareon P, Tomaniak M, Kawashima H, Takahashi K, Kogame N, Modolo R, Hara H, Gao C, Wang R, Walsh S, Suryapranata H, da Silva PC, Cotton J, Koning R, Akin I, Rensing B, Garg S, Wykrzykowska JJ, Piek JJ, Juni P, Hamm C, Steg PG, Valgimigli M, Windecker S, Storey RF, Onuma Y, Vranckx P, Serruys PW (2020) The association of body mass index with long-term clinical outcomes after ticagrelor monotherapy following abbreviated dual antiplatelet therapy in patients undergoing percutaneous coronary intervention: a prespecified sub-analysis of the GLOBAL LEADERS Trial. Clin Res Cardiol 109:1125-1139

5. Schupke S, Neumann FJ, Menichelli M, Mayer K, Bernlochner I, Wohrle J, Richardt G, Liebetrau C, Witzenbichler B, Antoniucci D, Akin I, Bott-Flugel L, Fischer M, Landmesser U, Katus HA, Sibbing D, Seyfarth M, Janisch M, Boncompagni D, Hilz R, Rottbauer W, Okrojek R, Mollmann H, Hochholzer W, Migliorini A, Cassese S, Mollo P, Xhepa E, Kufner S, Strehle A, Leggewie
S, Allali A, Ndrepepa G, Schuhlen H, Angiolillo DJ, Hamm CW, Hapfelmeier A, Tolg R, Trenk D, Schunkert H, Laugwitz KL, Kastrati A, Investigators I-RT (2019) Ticagrelor or Prasugrel in Patients with Acute Coronary Syndromes. N Engl J Med 381:1524-1534

6. Mayer K, Bongiovanni D, Karschin V, Sibbing D, Angiolillo DJ, Schunkert H, Laugwitz KL, Schupke S, Kastrati A, Bernlochner I (2020) Ticagrelor or Prasugrel for platelet inhibition in acute coronary syndrome patients: the ISAR-REACT 5 Trial. J Am Coll Cardiol 76:2569-2571

7. Dubois D, Dubois EF (1916) A formula to estimate the approximate surface area if height and weight be known. Arch Intern Med 17:863-871

8. DHSS/MRC Group on Obesity Research, James WP, Waterlow JC (1976) Research on obesity: a report of the DHSS/MRC Group

9. Nadler SB, Hidalgo JH, Bloch T (1962) Prediction of blood volume in normal human adults. Surgery 51:224-232 